\title{
Phân tích dòng thấm sử dụng môi trường ngẫu nhiên qua đập đất đá và bằng phương pháp phần tử hữu hạn
}

\author{
Võ Thị Tuyết Giang ${ }^{1,2}$, Nguyễn Võ Trọng ${ }^{1,2}$ \\ ${ }^{1}$ Khoa Kỹ Thuật Xây Dựng, Trường Đại học Bách khoa TP HCM, Đại học Quốc Gia TP HCM \\ ${ }^{2}$ Đại học Quốc gia Thành phố Hồ Chí Minh
}

\section{TỪ KHOÁ}

Phân tích thấm

Đập đất đá

Phương pháp phần tử hữu hạn

Hệ số thấm

Tính không chắc chắn

\begin{abstract}
TÓM TẮT
Bài báo này trình bày việc phân tích dòng thấm phát sinh trong ba loại đập đất được sử dụng phổ biến (đập đồng chất, đập có lõi giữa chống thấm và đập có lõi nghiêng chống thấm), trong đó hệ số thấm của đất đắp đập được xem xét có tính chất phân bố ngẫu nhiên theo không gian. Nghiên cứu tập trung đánh giá hai đại lượng quan trọng trong bài toán thấm là tổng lưu lượng thấm qua đập và gradient thấm. Cụ thể hơn, phương trình thấm qua đập đất được giải bằng phương pháp phần tử hữu hạn thông qua phần mềm tính thấm phổ biến hiện nay là SEEP/W. Hệ số thấm được phát sinh ngẫu nhiên thông qua hàm mật độ xác suất với giá trị trung bình và hệ số biến thiên tương ứng. Việc phát sinh giá trị ngẫu nhiên được mã hóa thông qua ngôn ngữ $\mathrm{C \#}$ và được tích hợp vào SEEP/W. Tiếp theo, Mô phỏng Monte Carlo được sử dụng kết hợp với các công cụ thống kê để tổng hợp và đánh giá kết quả. Các kết quả này được so sánh với các kết quả được tính toán từ phương pháp tất định. Theo đó, trong cả ba loại đập, có đến $100 \%$ khả năng lưu lượng tính toán tử phương pháp ngẫu nhiên lớn hơn lưu lượng tính toán từ phương pháp tất định. Trong khi đó, khoảng 50 \% khả năng gradient thấm tính toán từ phương pháp ngẫu nhiên lớn hơn gradient thấm tính toán từ phương pháp tất định. Các phân bố tần suất của lưu lượng phù hợp với phân bố chuẩn, trong khi đó các phân bố tần suất của gradient thấm có xu hướng lệch phải.
\end{abstract}

\section{KEYWORDS}

Seepage analysis

Earth-fill or rock-fill dams

Finite element method

Hydraulic conductivity

Uncertainty

\begin{abstract}
This paper presents the seepage analysis through three popular types of earth-fill dams (homogeneous, central-core and inclined core), whose hydraulic conductivities are considered as spatially random. This study focuses on evaluating two important parameters of seepage which are the flow rate and gradient. More specifically, the governing equation of seepage through earth-fill dams is solved using finite element analysis within a popular software, i.e. SEEP/W. The hydraulic conductivies are randomly generated based on the relevant probability density function with its mean and coefficient of variation. The generation of the random numbers is coded using C\# and integrated into SEEP/W. Next, the Monte Carlo simulations are applied, including statistical techniques for assessing the results. These outcomes then are compared with ones resulted from the determisnistic calculations. Hence, within all three types of dams, there is a probability of $100 \%$ in which the flow rates calculated from the random method are greater than ones from the deterministic method. As a meanwhile, there is a probability of about $50 \%$ in which the gradients calculated from the random method are greater than ones from the deterministic method. The frequency distributions of the flow rates fit well the normal distributions meanwhile the ones of the gradients show a right-skewed trend.
\end{abstract}

\section{Giới thiệu}

Phân tích thấm luôn được sử dụng trong quá trình thiết kế đập đất đá sử dụng vật liệu địa phương vì như đã nghiên cứu trong [1], người ta thấy một tỉ lệ lớn các vụ hư hỏng đập đất đá là do dòng thấm gây ra. Tuy nhiên, theo [2], trong quá trình phân tích dòng thấm, các phương pháp sử dụng tính tất định (deterministic) của các thông số tính toán như hệ số thấm sẽ gây ra ít nhiều sai lệch trong kết quả và trường phân bố ngẫu nhiên đã được đề xuất sử dụng để phân tích.
Việc sử dụng tính ngẫu nhiên của các thông số đất đá đã được sử dụng nhiều trong thời gian gần đây. Cụ thể hơn, có các nghiên cứu liên quan như phân tích trong bài toán cố kết (consolidation) của [3], đánh giá sức chịu tải của móng của [4], phân tích bài toán ổn định mái dốc của [5] hay phân tích dòng thấm của [6],[7]. Trong các nghiên cứu này, tính không chắc chắn của các thông số đất như hệ số thấm (hydraulic conductivity), sức chịu cắt không thoát nước (undrained shear strength), hay các thông số khác được xem xét. Ngoài ra còn có các nghiên cứu liên quan từ [8 - 16]. 
Một nghiên cứu khác đáng được quan tâm từ tài liệu [25]. Đây là một nghiên cứu có xét tính bất định của hệ số thấm khi xem xét bài toán thấm qua đập đất. Nghiên cứu này sử dụng phương pháp sai phân hữu hạn (finite difference method) và mục tiêu là đánh giá vị trí của đường bão hòa (free surface) trong thân đập. Nghiên cứu này cũng đã cho thấy, do tính bất định của hệ số thấm nên vị trí của mặt bão hòa trong thân đập cũng biến thiên, đặc biệt tại vị trí mặt hạ lưu.

Bên cạnh đó, SEEP/W là một phần mềm được sản xuất bởi Công ty GEO-SLOPE International Ltd. có trụ sở tại Canada [17]. Phần mềm này được sử dụng để thực hiện các phân tích hiện tượng thấm diễn ra trong môi trường đất. Phần mềm sử dụng công cụ toán số là phương pháp phần tử hữu hạn và có thể mô phỏng bài toán ổn định (steadystate) hoặc bài toán theo thời gian (transient).

Trong các phương pháp sử dụng, một phương pháp được sử dụng gần đây trong nghiên cứu của [6] và [7] có các ưu điểm như tích hợp phương pháp phần tử hữu hạn trong SEEP/W, một phần mềm khá phổ biến hiện nay trong phân tích thấm, và một giải thuật phát sinh trường ngẫu nhiên khá đơn giản so với các giải thuật khác. Do đó, bài báo sử dụng phương pháp này để phân tích dòng thấm có xét tính bất định của hệ số thấm vào việc xem xét các dạng đập đất đá khác nhau. Nghiên cứu sử dụng ba dạng đập đất đá phổ biến có hình dạng bên ngoài giống nhau, tuy nhiên phân bố bên trong có sự thay đổi. Cụ thể, đập đồng chất, đập có lõi giữa chống thấm và đập có lõi nghiêng chống thấm sẽ được sử dụng. Mục đích là để xem xét cùng một phương pháp, sự khác nhau (nếu có) ứng với các hình thức đập sẽ được phân tích và trình bày.

\section{Mô tả lý thuyết và phương pháp nghiên cứu}

Phương pháp nghiên cứu của bài báo này được tổ hợp từ các ý tưởng sau:

Lý thuyết thấm được mô tả qua phương trình chủ đạo (1).

Áp dụng lời giải toán số cho phương trình (1), cụ thể là phương pháp phần tử hữu hạn được thực hiện thông qua phần mềm SEEP/W.

Trong phương trình (1), do hệ số thấm $\mathrm{kx}$ và ky có xét giá trị trong vùng không bão hòa nên lý thuyết của van Genuchten [18] được sử dụng để mô tả đặc tính này.

Bên cạnh đó, do xem xét tính biến đổi (tính không chắn chắc) của hệ số thấm kx và ky nên các phương trình từ (3)-(7) được áp dụng.

Ngôn ngữ lập trình C\# được sử dụng để mã hóa các phương trình (3)-(7) và tích hợp vào phần mềm $S E E P / W$. Công cụ và cách thức để thực hiện việc mã hóa, người đọc có thể tham khảo tài liệu [26].

Thực hiện Mô phỏng Monte-Carlo với số lượng mẫu là 500 mẫu cho mỗi một mô phỏng.

Sử dụng các công cụ thống kê để đánh giá kết quả và so sánh với kết quả tính toán đến từ phương pháp tất định (tức phương pháp xem xét hệ số thấm là không thay đổi theo không gian).
Nội dung tiếp theo được triển khai để làm rõ thêm các phương pháp nghiên cứu này.

Bài toán dòng thấm ổn định (steady state) sử dụng phương trình chủ đạo sau [17]:

$$
\frac{\partial}{\partial x}\left(k_{x} \frac{\partial H}{\partial x}\right)+\frac{\partial}{\partial y}\left(k_{y} \frac{\partial H}{\partial y}\right)+Q=0
$$

trong đó, $\mathrm{H}$ là cột nước; $k_{x}$ và $k_{y}$ là hệ số thấm theo phương $\mathrm{x}$ và $\mathrm{y}$ (do bài toán xem xét môi trường đẳng hướng nên $k_{x}=k_{y}$ ); $Q$ là lưu lượng tại các biên.

Phương pháp phần tử hữu hạn được sử dụng để giải phương trình trên thông qua SEEP/W. Lưới phần tử trong SEEP/W được cung cấp gồm có loại tam giác (triangle), tứ giác (quadrilateral) hoặc hỗn hợp của hai dạng trên. Ngoài ra, khi tính toán các tích phân số (numerical integration), phần mềm cung cấp dạng 4 và 9 điểm Gauss cho phần tử tứ giác, 1 và 3 cho phần tử tam giác.

Do dòng thấm qua đập đất đá sẽ phát sinh vùng không bão hòa nên hệ số thấm được xem xét các giá trị trong khu vực không bão hòa. Hiện nay, có nhiều mô hình tính toán hệ số thấm có kể đến giá trị không bão hòa. Tuy nhiên, bài nghiên cứu này sử dụng mô hình được để xuất bởi [18] do tính phổ biến của nó, cụ thể

$$
K(h)=K_{s} K_{r}(h)
$$

trong đó, $K_{s}$ là hệ số thấm ở trạng thái bão hòa; $K_{r}(h)$ là dạng chuẩn hóa (normalized) của hệ số thấm không bão hòa và $h$ là cột áp. Trong vùng bão hòa, do $h>0$ nên $K_{r}(h)=1$ và trong vùng không bão hòa, $K_{r}(h)<1 . K_{r}(h)$ được tính toán theo hai thông số chính của mô hình là $\alpha$ và $n[18]$.

Khi xem xét tính không chắc chắn, bên cạnh $K_{s}$, hai hệ số $\alpha$ và $n$ cũng được xem xét như là biến phân bố ngẫu nhiên. Tuy nhiên, do lớp không bão hòa bên trên lớp bão hòa có lưu lượng thấm nhỏ so với toàn bộ lưu lượng thấm và để giảm việc tính toán, nghiên cứu đề xuất chỉ xem xét tính ngẫu nhiên của hệ số thấm và xem $\alpha$ và $n$ là không đổi (tất định) cho cùng một loại đất. Việc phát sinh các giá trị ngẫu nhiên được thực hiện thông qua hàm mật độ xác suất (probability density function - PDF) với giá trị trung bình (mean) và hệ số biến thiên (coefficient of variation - COV). Hệ số thấm của đất được cho là tuân theo phân bố lognormal (xem [6] và [7]). Do đó, việc phát sinh trường ngẫu nhiên của kệ số thấm được triển khai dựa theo các công thức sau ( $K$ là hệ số thấm bão hòa):

$$
\begin{gathered}
\sigma_{\ln K}^{2}=\ln \left(1+\frac{\sigma_{K}^{2}}{\mu_{K}^{2}}\right) \\
\mu_{\ln K}=\ln \mu_{K}-\frac{1}{2} \sigma_{\ln K}^{2} \\
\quad K=\exp \left(\mu_{\ln K}+\sigma_{\ln K} r\right)
\end{gathered}
$$

trong đó, $r$ là số ngẫu nhiên được phát sinh dựa theo công thức sau [19]:

$$
r=\left(-2 \ln u_{1}\right)^{1 / 2} \sin \left(2 \pi u_{2}\right)
$$

hay

$$
r=\left(-2 \ln u_{1}\right)^{1 / 2} \cos \left(2 \pi u_{2}\right)
$$


trong đó, $u_{1}$ và $u_{2}$ là hai biến ngẫu nhiên độc lập phát sinh từ hàm phân bố đồng nhất (uniform density function) trong khoảng $(0,1)$. Theo đó, hệ số thấm ngẫu nhiên được phát sinh và gán vào các điểm Gauss tương ứng. Việc khai triển các công thức này vào $\mathrm{SEEP} / \mathrm{W}$ có thể thực hiện thông qua bất kỳ ngôn ngữ lập trình nào mà có thể phát sinh mã CLR. Kết quả phân tích được thực hiện theo ý tưởng Mô phỏng Monte Carlo (MCS), tức là một số lượng các mô phỏng tương tụ được thực hiện và sử dụng các kỹ thuật thống kê để phân tích các kết quả tương ứng. Nhóm tác giả đã sử dụng ngôn ngữ $\mathrm{C} \#$ để viết các đoạn mã và tương thích vào thư viện của SEEP/W để có thể thực hiện đúng các ý tưởng trên. Hình 1 trình bày sơ đồ khối mô tả giải thuật phát sinh giá trị ngẫu nhiên của hệ số thấm đến các nút Gauss tương ứng.

Mô phỏng Monte Carlo được sử dụng để xem xét bài toán này. Cụ thể hơn, theo tinh thần của mô phỏng Monte Carlo, nghiên cứu này tiến hành lặp lại liên tục các mẫu ngẫu nhiên để đạt được kết quả cuối cùng. Ứng với một mẫu, giải thuật phát sinh hệ số thấm ngẫu nhiên sẽ phân phối giá trị hệ số thấm trong toàn bộ vùng tính toán. Lúc này, bài toán thấm sẽ được giải theo các giá trị hệ số thấm đã được gán và điều kiện biên tương ứng. Kết quả tính toán sẽ cho giá trị các thông số lưu lượng và gradient thấm tương ứng. Ghi nhận kết quả với chuỗi thực hiện lặp lại liên tục. Các công cụ thống kê sẽ được sử dụng để phân tích các chuỗi số liệu này.

Việc xem xét kết quả đầu ra của tính toán là gradient thấm (độ dốc thủy lực) cũng tương ứng với việc xem xét vận tốc thấm (tác nhân gây xói đất) do gradient thấm và vận tốc thấm có mối liên hệ với nhau.

Ngoài ra, trong một nghiên cứu liên quan, nhóm tác giả đã có các lưu ý khi sử dụng phương pháp này như sau:

- Giải thuật phát sinh số ngẫu nhiên r (Công thức (6) và (7) được xem là hợp lý nếu phân bố tần suất của các số này phù hợp với phân bố chuẩn (normal distribution).

- Việc làm mịn kích thước lưới phần tử đem lại sự chính xác hơn về mặt toán số [20], tuy nhiên việc thay đổi này làm thay đổi bản chất đầu vào của môi trường ngẫu nhiên vì làm thay đổi mức biến thiên (scale of fluctuation). Do đó, nếu bài toán có xét đến mức biến thiên thì các giải thuật phát sinh trường ngẫu nhiên khác nên được áp dụng (ví dụ [21]). Tuy nhiên, các giải thuật này phức tạp hơn.

- Số lượng mẫu để thực hiện MCS cần đủ lớn để đem lại kết quả tin cậy. Tuy nhiên, việc này sẽ mất nhiều thời gian khi làm việc với SEEP/W. Để cân bằng giữa thời gian thực hiện và độ chính xác cần có, 500 mẫu là số có thể chấp nhận được.

Theo tinh thần của Mô phỏng Monte-Carlo, số lượng thực hiện mẫu càng nhiều thì kết quả càng tốt hơn. Tuy nhiên, do việc mô phỏng tốn khá nhiều thời gian nên nghiên cứu cố gắng tìm số lượng mẫu có thể chấp nhận được để cân bằng giữa tính hợp lý của kết quả và thời gian mô phỏng. Trong Mô phỏng Monte-Carlo, số lượng mẫu chấp nhận được khi các thông số thống kê của một mô phỏng (ví dụ giá trị trung bình mean, và hệ số biến thiên coefficent of variation - COV) đi đến ổn định. Theo đó, nghiên cứu đã chọn số lượng mẫu là 500 để đáp ứng yêu cầu trên. Số lượng 500 này cũng được sử dụng trong một số nghiên cứu được trích dẫn bên trên.

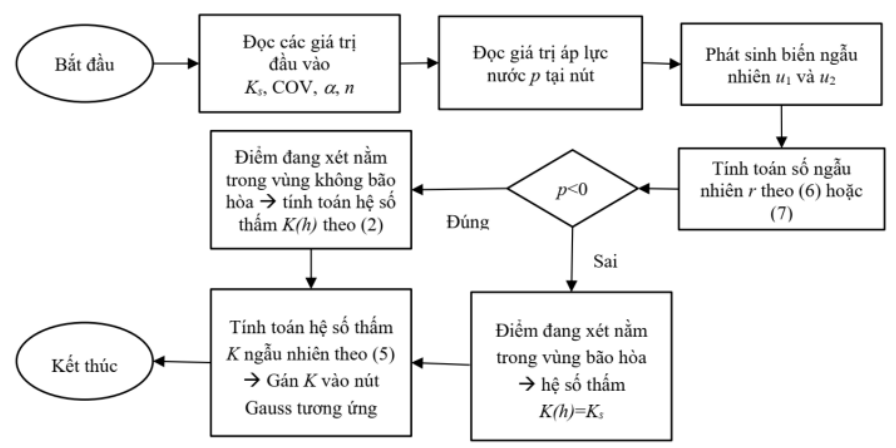

Hình 1. Sơ đồ khối mô tả việc phát sinh hệ số thấm ngẫu nhiên và được gán vào các nút Gauss tương ứng.

\section{3. Áp dụng vào các hình thức đập đất đá khác nhau}

Trên cơ sở đó, bài nghiên cứu này sử dụng phương pháp đã trình bày để phân tích dòng thấm qua các dạng đập đất đá khác nhau [22]. Cụ thể hơn, 3 dạng đập phổ biến được trình bày trong Hình 2 (đập đồng chất), Hình 3 (đập có phần lõi giữa chống thấm) và Hình 4 (đập có lõi chống thấm dạng lõi nghiêng). Mục đích của việc phân tích là xem xét sự tác động của môi trường ngẫu nhiên lên các dạng đập khác nhau. Do đó, vật liệu sử dụng giống nhau các thông số như $\mathrm{COV}$, a và $\mathrm{n}$ và được trình bày trong Bảng 1 . Các thông số này được sử dụng dựa vào các thống kê nghiên cứu liên quan từ tài liệu [27]. Các giá trị kết quả dùng để phân tích là tổng lưu lượng thấm qua đập và gradient thấm tại các vị trí quan trọng ứng với từng loại đập (xem Hình 2 , Hình 3 và Hình 4 để biết các vị trí cần xem xét gradient thấm). Các giá trị gradient (độ dốc thủy lực) được xem xét là giá trị cục bộ tại vị trí đang xem xét được xuất ra thông qua SEEP/W.

Cần lưu ý thêm, ba loại đập sử dụng trong nghiên cứu là các hình thức đập thông dụng khi xây dựng đập đất sử dụng vật liệu tại chỗ. Tùy vào điều kiện sẵn có của vật liệu và điều kiện thi công cũng như yêu cầu của công trình, một hình thức đập cụ thể sẽ được chọn. Thông thường, khi đập có lõi chống thấm thì vật liệu sẽ có hệ số thấm thấp hơn các phần đắp khác (được thể hiện qua Bảng 1). Như vậy, sự khác nhau ở ba loại đập chính là hình thức cấu tạo (có hay không có lõi chống thấm; hình dạng lõi chống thấm như thế nào, nghiêng hay trung tâm...).

Lưới phần tử dùng để phân tích là phần tử tam giác (triangle) với 3 điểm Gauss. Kích thước phần tử trung bình được sử dụng là 2,5 m. Kích thước này được sử dụng trên cơ sở lưới tối ưu của SEEP/W dựa trên kích thước tồng thể của các đập đưa vào. Lưới có thể được mịn hơn để có kết quả tốt hơn về mặt toán số tuy nhiên lúc này lại gặp vấn đề về mức biến thiên (scale of fluctuation) của trường ngẫu nhiên. Nếu mức biến thiên không được xem xét, bài nghiên cứu sử dụng kích thước lưới tối ưu mà SEEP/W cung cấp (tức $2,5 \mathrm{~m}$ ). Theo khẳng định từ tài liệu tham khảo [17], lưới này đã được tối ưu và chấp nhận được về tính hợp lý với một miền tính toán bất kỳ. Lưới được đánh giá có mức độ mịn dạng trung bình (medium). 


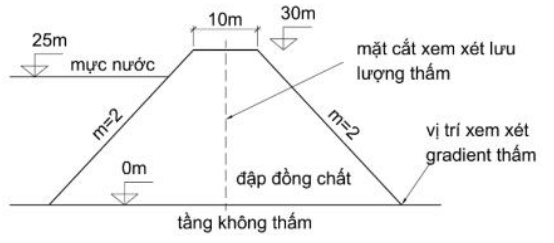

Hình 2. Hình dạng đập đất đồng chất sử dụng để phân tích.

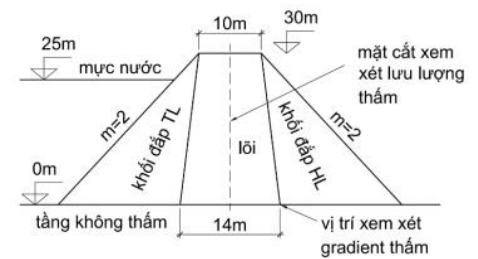

Hình 3. Hình dạng đập đất có lõi giữa sử dụng để phân tích.

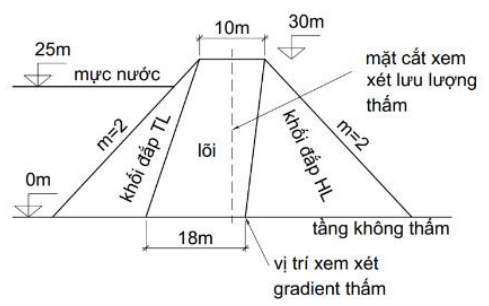

Hình 4. Hình dạng đập đất có lõi nghiêng sử dụng để phân tích.

\subsection{Số ngẫu nhiên và sự phân bố tương ứng}

Như đã trình bày, số ngẫu nhiên $r$ đóng vai trò quan trọng của phương pháp này. Do đó, phần này trình bày sự phân bố số $\mathrm{r}$ trong thân đập. Các Hình 5 , Hình 6 , Hình 7 , Hình 8 , Hình 9 và Hình 10 trình bày phân bố của số ngẫu nhiên trong toàn bộ thân đập cũng như các bộ phận (lõi, phần đắp thượng lưu, phần đắp hạ lưu) của 3 loại đập.

Bảng 1.

Các thông số đầu vào.

\begin{tabular}{|c|c|c|}
\hline Thông số & Phần đất đắp & Phần lõi \\
\hline Trung bình của hệ số thấm & $1 \times 10^{-6}(\mathrm{~m} / \mathrm{s})$ & $1 \times 10^{-8}(\mathrm{~m} / \mathrm{s})$ \\
\hline COV của hệ số thấm & 2 & 2 \\
\hline $\begin{array}{c}\alpha \text { (mô hình van } \\
\text { Genuchten) }\end{array}$ & 0,02 & 0,02 \\
\hline $\begin{array}{l}n \text { (mô hình van } \\
\text { Genuchten) }\end{array}$ & 1,40 & 1,40 \\
\hline & & 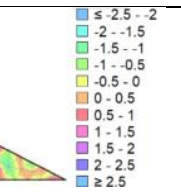 \\
\hline
\end{tabular}

Hình 5. Sự phân bố của số ngẫu nhiên $\mathrm{r}$ của một mẫu trong toàn bộ thân đập ở dạng contour - đập đồng chất.

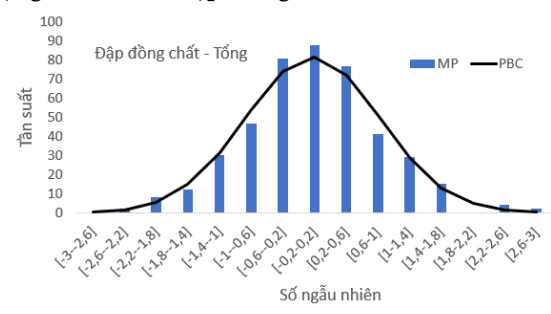

Hình 6. Phân bố số ngẫu nhiên $\mathrm{r}$ của một mẫu trong toàn bộ thân đập đồng chất (MP-Mô phỏng; PBC-Phân bố chuẩn).

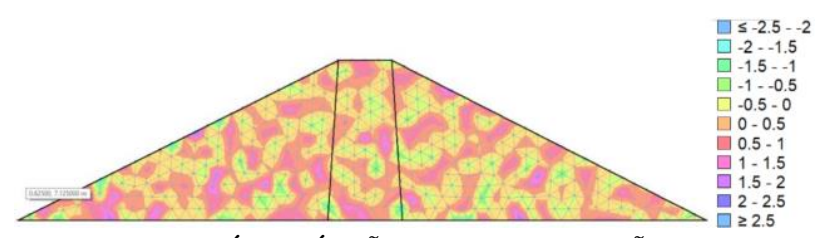

Hình 7. Sự phân bố của số ngẫu nhiên $\mathrm{r}$ của một mẫu trong toàn bộ thân đập ở dạng contour - đập có lõi giữa.

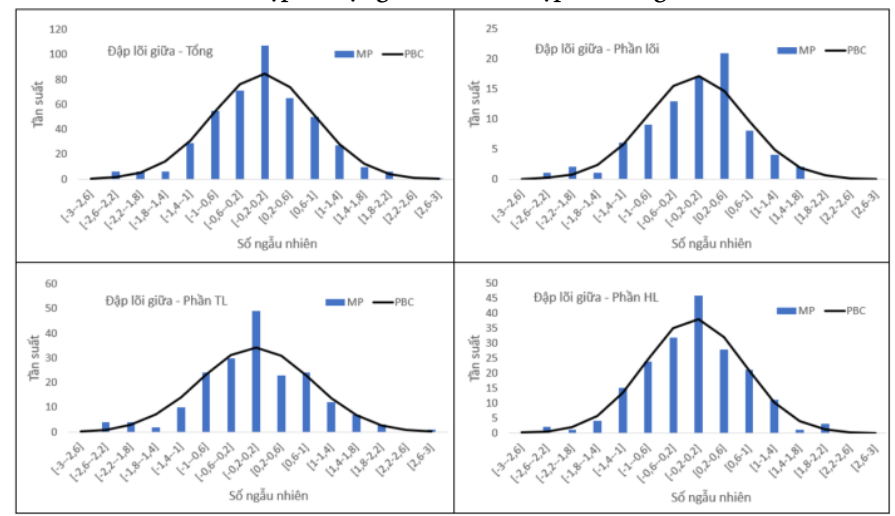

Hình 8. Phân bố số ngẫu nhiên $r$ của một mẫu trong toàn bộ thân đập, phần lõi, phần đắp thượng lưu và phần đắp hạ lưu của đập có lõi giữa (MP-Mô phỏng; PBC-Phân bố chuẩn).

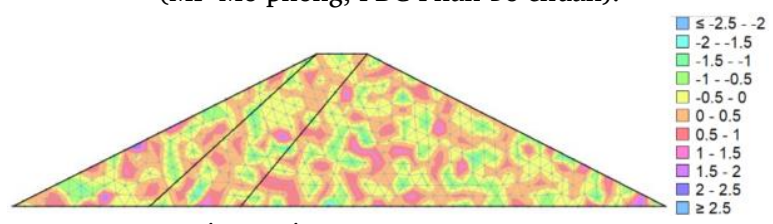

Hình 9. Sự phân bố của số ngẫu nhiên $r$ của một mẫu trong toàn bộ thân đập ở dạng contour - đập có lõi nghiêng.

Bảng 2.

Kiểm định Chi bình phương với các phân bố số ngẫu nhiên r.

\begin{tabular}{ccc}
\hline Phân bố & $\chi_{\text {calculated }}^{2}$ & $\chi_{\text {limit }}^{2}$ \\
\hline Đập đồng chất (toàn bộ) & 5,6 & 18,3 \\
\hline Đập lõi giữa (toàn bộ) & 19,5 & 18,3 \\
\hline Đập lõi giữa (phần lõi) & 4,4 & 12,6 \\
\hline Đập lõi giữa (phần TL) & 10,0 & 15,5 \\
\hline Đập lõi giữa (phần HL) & 4,9 & 15,5 \\
\hline $\begin{array}{c}\text { Đập lõi nghiêng (toàn } \\
\text { bộ) }\end{array}$ & 3,9 & 16,9 \\
\hline $\begin{array}{c}\text { Đập lõi nghiêng (phần } \\
\text { lõi) }\end{array}$ & 5,5 & 12,6 \\
\hline $\begin{array}{c}\text { Đập lõi nghiêng (phần } \\
\text { TL) }\end{array}$ & 7,0 & 12,6 \\
\hline Đập lõi nghiêng (phần \\
HL)
\end{tabular}

Theo lý thuyết, phân bố tần suất của số ngẫu nhiên $\mathrm{r}$ phải tuân thủ theo phân bố chuẩn (normal distribution). Các Hình 6 , Hình 8 và Hình 10 thể hiện các đường phân bố chuẩn về mặt lý thuyết được cho là mô tả tốt nhất các phân bố tần suất này. Để đánh giá có tính định lượng hơn, nghiên cứu đã sử dụng Kiểm định Chi bình phương (Chisquared Test) được tham khảo từ tài liệu [28] để đánh giá mức độ phù hợp (goodness of fit) của các phân phối này. 
Bảng 3.

Số liệu thống kê của MCS.

\begin{tabular}{|c|c|c|c|c|c|c|c|c|c|}
\hline \multirow[t]{2}{*}{ Dạng } & \multicolumn{4}{|c|}{ Tổng lưu lượng thấm } & \multicolumn{5}{|c|}{ Gradient thấm } \\
\hline & $\begin{array}{c}\text { Trung bình } \\
\text { (Mean) } \\
\left(\times 10^{7} \mathrm{~m}^{3} / \mathrm{s}\right)\end{array}$ & $\begin{array}{c}\mathrm{SD} \\
\left(\times 10^{7} \mathrm{~m}^{3} / \mathrm{s}\right)\end{array}$ & $\mathrm{COV}$ & $\begin{array}{l}\text { Deter. } \\
\left(\times 10^{7}\right. \\
\left.\mathrm{m}^{3} / \mathrm{s}\right)\end{array}$ & $\begin{array}{l}\text { Trung } \\
\text { bình } \\
\text { (Mean) }\end{array}$ & SD & $\mathrm{COV}$ & $\begin{array}{c}\text { Độ lệch } \\
\text { (Skewness) }\end{array}$ & Deter. \\
\hline Đồng chất & 74,005 & 3,005 & 0,041 & 41,560 & 0,509 & 0,038 & 0,074 & 0,291 & 0,508 \\
\hline Lõi giữa & 4,400 & 0,415 & 0,094 & 2,458 & 0,507 & 0,206 & 0,406 & 0,508 & 0,542 \\
\hline \multicolumn{10}{|c|}{ Chú thích: } \\
\hline SD & sta & rd deviation $(\mathrm{c}$ & lệch ch & & & & & & \\
\hline $\mathrm{COV}$ & \multicolumn{9}{|c|}{ coefficient of variation (hệ số biến thiên) } \\
\hline Deter. & \multicolumn{9}{|c|}{ deterministic (kết quả tính theo giá trị tất định) } \\
\hline
\end{tabular}

Bảng 4.

Kiểm định Chi bình phương với các phân bố của lưu lượng và gradient thấm.

\begin{tabular}{ccccc}
\hline \multirow{2}{*}{ Dạng đập } & \multicolumn{2}{c}{ Lưu lượng } & \multicolumn{2}{c}{ Gradient thấm } \\
\cline { 2 - 5 } & $\chi_{\text {calculated }}^{2}$ & $\chi_{\text {limit }}^{2}$ & $\chi_{\text {calculated }}^{2}$ & 40,9 \\
\hline Đập đồng chất & 13,5 & 22,4 & 40,9 & 27,6 \\
\hline Đập lõi giữa & 20,3 & 30,1 & 15,9 & 28,9 \\
\hline Đập lõi nghiêng & 31,1 & 37,7 & 33,9 \\
\hline
\end{tabular}

Bảng 5.

Đánh giá xác suất giá trị tính toán từ phương pháp ngẫu nhiên lớn hơn giá trịnh tính toán tử phương pháp tất định.

\begin{tabular}{ccc}
\hline Dạng đập & Lưu lượng & Gradient thấm \\
\hline Đập đồng chất & $100 \%$ & $51 \%$ \\
\hline Đập lỗi giữa & $100 \%$ & $43 \%$ \\
\hline Đập lõi nghiêng & $100 \%$ & $49 \%$ \\
\hline
\end{tabular}

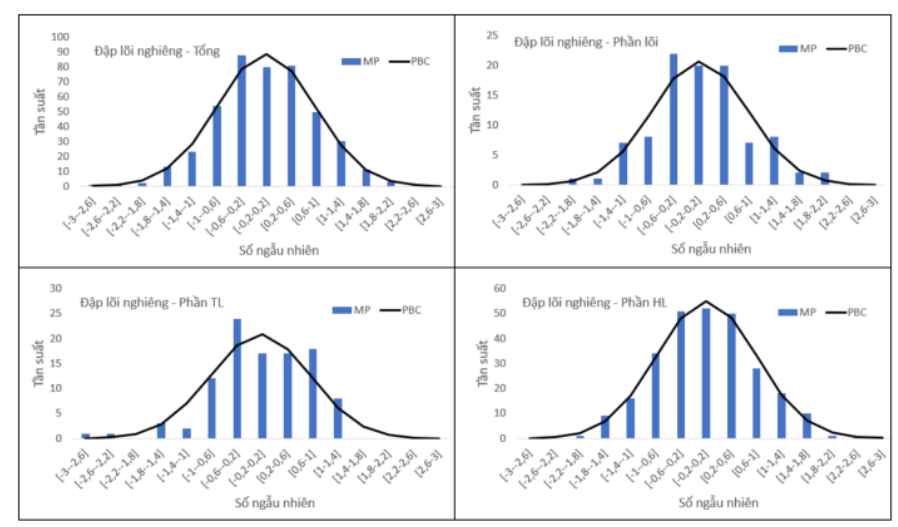

Hình 10. Phân bố số ngẫu nhiên $r$ của một mẫu trong toàn bộ thân đập, phần lõi, phần đắp thượng lưu và phần đắp hạ lưu của đập có lõi nghiêng (MP-Mô phỏng; PBC-Phân bố chuẩn).

Kiểm định này sử dụng một thông số được gọi là Chi bình phương tính toán ( $\chi_{-}$calculated`2) và được tính toán dựa vào sự khác biệt giữa phân phối tần suất thực tế và phân bố chuẩn tương ứng. Bên cạnh đó, giá trị Chi bình phương giới hạn ( $\left.\chi_{\sim} \operatorname{limit}^{\wedge} 2\right)$ cũng được tính toán với ý nghĩa thống kê (level of significance) $5 \%$ được sử dụng để so sánh. Nếu $\chi_{-}$calculated` $2<\chi_{\sim} \operatorname{limit}^{\wedge} 2$, phân bố chuẩn lý thuyết được cho là phù hợp với phân bố tuần suất từ thí nghiệm. Bảng 2 trình bày các so sánh từ Hình 6 , Hình 8 và Hình 10 . Kết quả cho thấy chỉ duy

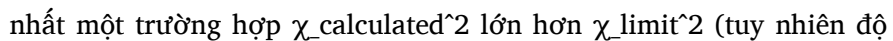
chênh lệch cũng không nhiều), tất cả các trường hợp còn lại cho thây giải thuật phát sinh số ngẫu nhiên $r$ cùng kích cỡ lưới sử dụng đã đáp ứng với yêu cầu đã nêu ra.

\subsection{Kết quả sử dụng MCS}

Với hình dạng và các số liệu đầu vào như trên, ứng với mỗi dạng đập, MCS được thực hiện với 500 mẫu. Kết quả được thể hiện trong các Hình 11 , Hình 12 và Hình 13 và Bảng 3 , Bảng 4 và Bảng 5 .

Từ các kết quả trên, nghiên cứu đã có các nhận xét sau:

- Với các hình dạng đập có cấu tạo phức tạp hơn (có lõi chống thấm), mức độ biến động của kết quả (thông qua thông số COV) có giá trị lớn hơn (ví dụ, với lưu lượng thấm, $\mathrm{COV}$ của đập có lõi là 0,094 và 0,087 so với 0,041 của đập đồng chất; với gradient thấm, COV của đập có lõi là 0,406 và 0,300 so với 0,074 của đập đồng chất) (Bảng 3). 
- Sử dụng hàm phân bố để tính toán, nghiên cứu cho thấy có đến 100 \% xác suất lưu lượng tính từ phương pháp ngẫu nhiên lớn hơn lưu lượng tính từ phương pháp tất định. Bên cạnh đó, có đến khoảng 50 \% khả năng gradient thấm tính từ phương pháp ngẫu nhiên lớn hơn gradient thấm tính từ phương pháp tất định (Bảng 5).

- Sử dụng Kiểm định Chi bình phương, nghiên cứu cho thấy phân bố tuần suất của lưu lượng phù hợp với phân bố chuẩn tương ứng (do $\chi_{\text {calculated }}^{2}<\chi_{\text {limit }}^{2}$ ). Trong khi đó, hai trong ba trường hợp của gradient thấm, phân bố tần suất chưa phù hợp hoàn toàn với phân bố chuẩn tương ứng (Bảng 4). Tính toán thêm hệ số lệch (skewness) của các phân bố gradient này (Bảng 3 ) cho thấy các phân bố có xu hướng lệch phải.

\section{Bảng 6.}

Các thông số liên quan đến một ví dụ đập đá đổ có lõi chống thấm và bộ phận chuyển tiếp.

\begin{tabular}{|c|c|c|c|c|c|c|c|c|c|c|c|c|c|c|}
\hline \multicolumn{2}{|c|}{ Lõi giữa } & \multicolumn{4}{|c|}{ Lớp lọc 1} & \multicolumn{4}{|c|}{ Lớp lọc 2} & \multicolumn{4}{|c|}{ Lớp lọc 3} & \multirow{2}{*}{$\begin{array}{c}\text { Đá đổ } \\
\mathrm{D} \\
(\mathrm{mm})\end{array}$} \\
\hline $\begin{array}{c}\mathrm{D}_{50} \\
(\mathrm{~mm})\end{array}$ & $\begin{array}{c}D_{85} \\
(\mathrm{~mm})\end{array}$ & $\begin{array}{c}\mathrm{D}_{15} \\
(\mathrm{~mm})\end{array}$ & $\begin{array}{c}\mathrm{D}_{50} \\
(\mathrm{~mm})\end{array}$ & $\begin{array}{c}\mathrm{D}_{85} \\
(\mathrm{~mm})\end{array}$ & $\begin{array}{l}\text { Bề } \\
\text { dày } \\
\text { (m) }\end{array}$ & $\begin{array}{c}\mathrm{D}_{15} \\
(\mathrm{~mm})\end{array}$ & $\begin{array}{c}D_{50} \\
(\mathrm{~mm})\end{array}$ & $\begin{array}{c}D_{85} \\
(\mathrm{~mm})\end{array}$ & $\begin{array}{l}\text { Bề } \\
\text { dày } \\
\text { (m) }\end{array}$ & $\begin{array}{c}\mathrm{D}_{15} \\
(\mathrm{~mm})\end{array}$ & $\begin{array}{c}\mathrm{D}_{50} \\
(\mathrm{~mm})\end{array}$ & $\begin{array}{c}\mathrm{D}_{85} \\
(\mathrm{~mm})\end{array}$ & $\begin{array}{l}\text { Bề } \\
\text { dày } \\
\text { (m) }\end{array}$ & \\
\hline 0,01 & 0,06 & 0,5 & 1,0 & 1,5 & 0,5 & 6 & 12 & 18 & 1 & 70 & 140 & 200 & 3 & 500 \\
\hline
\end{tabular}

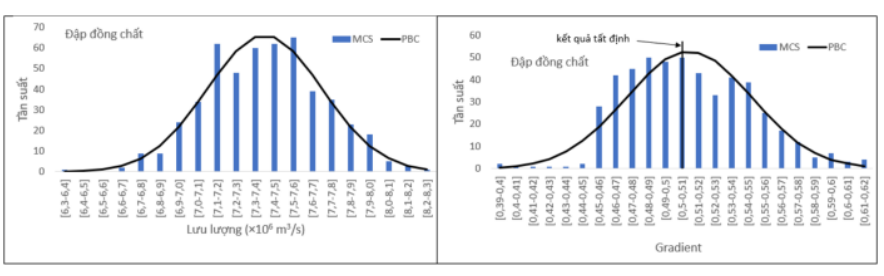

Hình 11. Kết quả của MCS trong đập đồng chất của tổng lưu lượng thấm và gradient thấm (MCS-Mô phỏng Monte Carlo; PBC-Phân bố chuẩn).

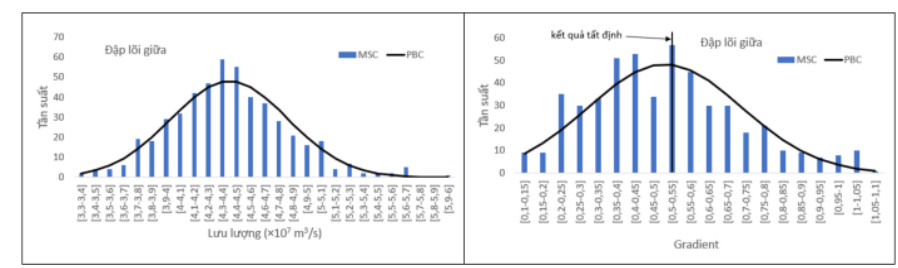

Hình 12. Kết quả của MCS trong đập có lõi giữa của tổng lưu lượng thấm và gradient thấm (MCS-Mô phỏng Monte Carlo; PBC-Phân bố chuẩn).

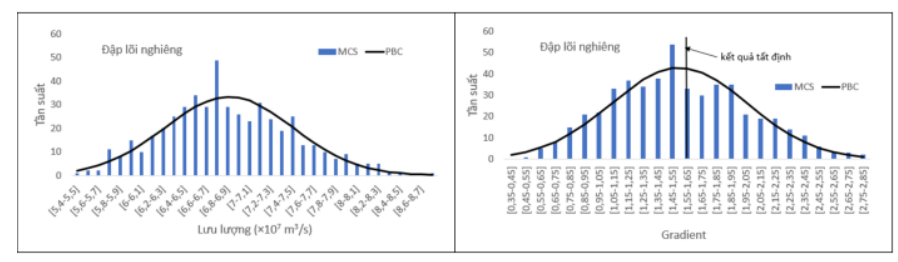

Hình 13. Kết quả của MCS trong đập có lõi nghiêng của tổng lưu lượng thấm và gradient thấm (MCS-Mô phỏng Monte Carlo; PBC-Phân bố chuẩn).

\section{3. Áp dụng trong đập đá đổ có lõi chống thấm ở giữa}

Phần này chỉ trình bày các ý tưởng khi mở rộng việc áp dụng cho các hình thức đập đất đá khác, ví dụ như đập đá đổ khi bộ phận chuyển tiếp giữa lõi và phần đắp thượng và hạ lưu phức tạp hơn.

Việc áp dụng có thể được mở rộng khi phân tích một đập đá đổ có lõi chống thấm ở giữa. Một ví dụ sử dụng đất mịn làm lõi chống thấm và phần đá đổ có kích thước đá lớn nên để ngăn ngừa xói trong lõi, một bộ phận chuyển tiếp được xây dựng kèm theo. Theo tiêu chuẩn thiết kế các lớp lọc của [23] và [24], kích thước có thể của các lớp lọc được trình bày như theo Hình 14 và Bảng 6 . Theo mô tả lý thuyết của phần mềm SEEP/W trong tài liệu [17], với các vật liệu có kích cỡ hạt như lớp lọc 3 trở lên thì vật liệu này về mặt vật lý không có ý nghĩa trong việc tiêu tán năng lượng thấm (cột nước) nên vùng này không cần mô tả và mô phỏng trong bài toán thấm. Do đó, mô phỏng chỉ cần xét phần lõi, lớp lọc 1 và lớp lọc 2 . Các công việc như phát sinh hệ số thấm ngẫu nhiên và sử dụng MCS vẫn được thực hiện như đã mô tả bên trên.

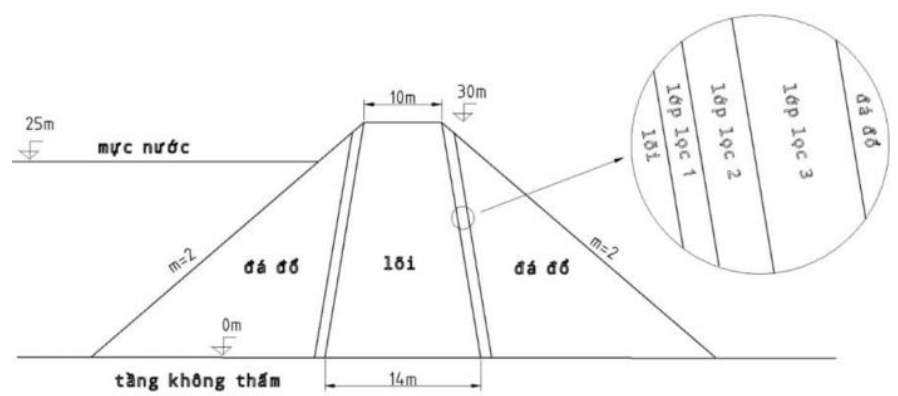

Hình 14. Hình dạng đập đá đổ có lõi chống thấm với bộ phận chuyển tiếp. 


\section{Kết luận}

Bài báo này trình bày một nghiên cứu liên quan đến sự phân tích hiện tượng thấm đi qua ba loại đập đất phổ biến hiện nay. Tuy nhiên, khác với các phân tích tất định khi hệ số thấm được xem xét như không thay đổi trong cùng một lớp đất, nghiên cứu này sử dụng tính ngẫu nhiên của hệ số thấm để xem xét bài toán.

Phương pháp nghiên cứu của bài báo này là kết hợp phương pháp phần tử hữu hạn thông qua phần mềm SEEP/W và xây dựng giải thuật phát sinh số ngẫu nhiên $r$, từ đó phát sinh hệ số thấm có sự phân bố ngẫu nhiên trong thân đập đất. Mô phỏng Monte Carlo được sử dụng để phân tích tính ngẫu nhiên của bài toán thông qua số lượng 500 mẫu cho một trường hợp phân tích. Các công cụ thống kê được sử dụng để phân tích các kết quả này.

Nghiên cứu đã cho thấy, giải thuật phát sinh số ngẫu nhiên $r$ được xây dựng hợp lý khi các phân bố tần suất của số ngẫu nhiên này trong thân đập phù hợp với phân bố chuẩn.

Bên cạnh đó, nghiên cứu cũng cho thấy với các đập có có lõi chống thấm, mức độ biến động của kết quả (thông qua thông số COV) có giá trị lớn hơn (ví dụ, với lưu lượng thấm, $\mathrm{COV}$ của đập có lõi là 0,094 và 0,087 so với 0,041 của đập đồng chất; với gradient thấm, $\mathrm{COV}$ của đập có lõi là 0,406 và 0,300 so với 0,074 của đập đồng chất).

Ngoài ra, nghiên cứu còn cho thấy có đến $100 \%$ xác suất lưu lượng tính từ phương pháp ngẫu nhiên lớn hơn lưu lượng tính từ phương pháp tất định và có khoảng 50 \% khả năng gradient thấm tính từ phương pháp ngẫu nhiên lớn hơn gradient thấm tính từ phương pháp tất định. Kết luận này rất quan trọng vì đã cho thấy việc thiết kế chỉ sử dụng kết quả tất định đã chưa lường hết các rủi ro do sự ngẫu nhiên của tính chất vật liệu gây ra.

Nghiên cứu còn cho thấy phân bố tuần suất của lưu lượng phù hợp với phân bố chuẩn tương ứng. Trong khi đó, với gradient thấm, phân bố tần suất chưa phù hợp hoàn toàn với phân bố chuẩn tương ứng và các phân bố có xu hướng lệch phải.

Tuy nhiên, nghiên cứu chưa đánh giá sự ảnh hưởng của sự thay đổi các thông số (ví dụ như COV) lên kết quả đầu ra. Đây cũng là dự kiến trong các nghiên cứu sắp tới của tập thể tác giả.

\section{Lời cảm ơn}

Nghiên cứu này được tài trợ bởi Đại học Quốc gia Thành phố Hồ Chí Minh (ĐHQG-HCM) trong khuôn khổ đề tài mã số C2020-20-21.

\section{Tài liệu tham khảo}

[1] Foster, M., Fell, R., Spannagle, M. (2000). The statistics of embankment dam failures and accidents. Can. Geotech. J., 37(5): 1000-1024.

[2] Fenton, G., Griffiths, D. (1996). Statistics of free surface flow through stochastic earth dam. J. Geotech. Eng., https://doi.org/10.1061/(ASCE)07339410(1996)122:6(427).

[3] Bari, M., Shahin, M., Nikraz, H. (2013). Probabilistic analysis of soil consolidation via prefabricated vertical drains. Int. J. Geomech, https://doi.org/10.1061/(ASCE)GM.1943-5622.0000244.
[4] Griffiths, D., Fenton, G., Manoharan, N. (2006). Undrained bearing capacity of twostrip footings on spatially random soil. Int. J. Geomech, 10.1061/(ASCE)15323641(2006)6:6(421).

[5] Genevois, R., Romeo, R. (2003). Probability of failure occurrence and recurrence in rock slopes stability analysis. Int. J. Geomech, https://doi.org/10.1061 /(ASCE)1532-3641.

[6] Calamak, M., Yanmaz, A. M. (2016). Uncertainty quantification of transient unsaturated seepage through embankment dams. Int. J. Geomech., https://doi.org/10.1061/(ASCE)GM.1943-5622.0000823.

[7] Calamak, M, Yanmaz, A. M. (2018). Assessment of core-filter configuration performance of rock-fill dams under uncertainties. Int. J. Geomech., 18(4), 15323641.

[8] Freeze, R. A. (1975). A stochastic-conceptual analysis of one-dimensional groundwater flow in nonuniform homogeneous media. Water Resour. Res., 11(5): 725-741.

[9] Gutjahr, A. L., Gelhar, L. W. (1981). Stochastic models of subsurface flow: Infinite versus finite domains and stationarity. Water Resour. Res., 17(2): 337-350.

[10] Lin, G. F., Chen, C. M. (2004). Stochastic analysis of spatial variability in unconfined groundwater flow. Stochastic Environ. Res. Risk Assess., 18(2): 100-108.

[11] Mantoglou, A., Gelhar, L. W. (1987). Stochastic modeling of largescale transient unsaturated flow systems. Water Resour. Res., 23(1): 37-46.

[12] Tartakovsky, D. M. (1999). Stochastic modeling of heterogeneous phreatic aquifers. Water Resour. Res., 35(12): 3941-3945.

[13] Zhang, D. (1999). Nonstationary stochastic analysis of transient unsaturated flow in randomly heterogeneous media. Water Resour. Res., 35(4): 1127-1141.

[14] Ahmed, A. A. (2009). Stochastic analysis of free surface flow through earth dams. Comput. Geotech., 36(7): 1186-190.

[15] Ahmed, A. A. (2013). Stochastic analysis of seepage under hydraulic structures resting on anisotropic heterogeneous soils. J. Geotech. Geoenviron. Eng., 139(6): 1001-1004.

[16] Le, T. M. H., Gallipoli, D., Sanchez, M., Wheeler, S. J. (2012). Stochastic analysis of unsaturated seepage through randomly heterogeneous earth embankments. Int. J. Numer. Anal. Methods Geomech, 36(8): 1056-1076.

[17] Geo-Slope International Ltd. (2012). Seepage modelling with SEEP/W. Calgary, Canada.

[18] van Genuchten, M. T. (1980). A closed-form equation for predicting the hydraulic conductivity of unsaturated soils. Soil Sci. Soc. Am. J., 44: 892-898.

[19] Box, G. E. P., Muller, M. E. (1958). A note on the generation of random normal deviates. Ann. Math. Stat., 29(2), 610-611.

[20] Hutton, D. V. (2004). Fundamentals of finite element analysis, $1^{\text {st }}$ edn. McGraw-Hill, New York.

[21] Fenton, G., Vanmarcke, E. H. (1990). Simulation of random fields via local average subdivision. J. Eng. Mech., 116(8): 1733-1749.

[22] Novak, P., Moffat, A.I.B, Nalurri, C., Narayanan, R. (2007). Hydraulic Structures, $4^{\text {th }}$ edn. Taylor and Francis, Abingdon.

[23] USDA (1994). Gradation design of sand and gravel filters, Chapter 26, Part 633. Nationnal Engineering Handbook.

[24] ICOLD (1994). Embankment dams granular filters and drains, Bulletin 95. CIGB ICOLD.

[25] Calamak, M., Kentel, E., Yanmaz, A.M. (2012). Seepage Analysis through Earth-fill Dams Having Random Fields. 10th International Congress on Advances in Civil Engineering, 17-19 October 2012. Middle East Technical University, Ankara, Turkey.

[26] Geo-Slope International Ltd. (2012). GeoStudio Add-Ins Programming Guide and Reference. Calgary, Canada.

[27] Carsel, R.F, Parrish, R.S. (1988). Developing joint probability distributions of soil water retention charasteristics. Water Resour. Res., 24(5): 755-769.

[28] DeCoursey, W.J. (2003). Statistics and Probability for Engineering Applications. Newnes. 